\title{
Favouring Responsible Publishing: A Protocol for Creating a Database of Researchers and Surveying Their Knowledge, Attitudes and Opinions towards Open Access Publishing and a New Field-Specific Journal
}

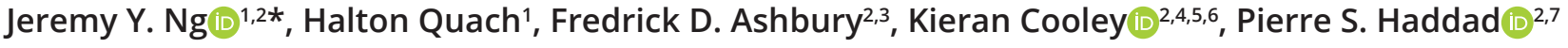 \\ ${ }^{1}$ Department of Health Research Methods, Evidence, and Impact, Faculty of Health Sciences, McMaster University, \\ Hamilton, Ontario, Canada \\ ${ }^{2}$ NHP Publications, Toronto, Ontario, Canada \\ ${ }^{3}$ Institute of Health Policy, Management and Evaluation, University of Toronto, Toronto, Ontario, Canada \\ ${ }^{4}$ Australian Research Center in Complementary and Integrative Medicine, University of Technology, Ultimo, Australia \\ ${ }^{5}$ Pacific College of Oriental Medicine, San Diego, California, United States of America \\ ${ }^{6}$ Department of Research, Canadian College of Naturopathic Medicine, Toronto, Ontario, Canada \\ ${ }^{7}$ Department of Pharmacology and Physiology, Faculty of Medicine, University of Montreal, Montreal, Quebec, Canada \\ *@ Corresponding Author: ngjy2@mcmaster.ca
}

\begin{abstract}
BACKGROUND: As a new publication, the Journal of Natural Health Product Research (INHPR) sought to develop a two-step protocol that (1) creates an international database of natural health product (NHP) researchers and (2) surveys their perceptions, needs, and preferences for a legitimate, broad-based, open access scientific journal in their field. This protocol could be useful to other researchers and publishers and can be adapted to establish similarly legitimate journals within their academic discipline.

METHODS: The database will initially be created using publicly available information online. A keyword-based, systematized search will identify the majority of NHP researchers, with referrals completing the process. Key research and publication characteristics of each person will be recorded. Subsequently, a survey will be developed and administered to all identified individuals to capture their attitudes and opinions on both open access in general, as well as the JNHPR. Construction of our NHP researcher database began in early-2018 and the development of our survey began in mid-2019. The survey is slated to be administered in early 2020. CONCLUSION: This protocol provides a process for constructing an international researcher database and a survey that aims to understand the community of NHP researchers' interests and needs regarding open access publishing and a new field-specific journal. This process can be modified and replicated for the purpose of establishing other legitimate journals in any scientific research discipline.
\end{abstract}

KEYWORDS: journalology; JNHPR; natural health product; natural product; NHP; open access; predatory publishing; protocol; publication science

\section{Background}

Open access is a model of publishing that allows readers to freely access literature written by others. The existence of open access journals in academia has rapidly increased alongside the surge of Internet usage for the dissemination of information [1]. Unfortunately, there is an increasing number of digitally based, open access journals that have exploited authors by charging them publication fees while providing poor editorial or publishing practices $[2,3]$. Such exploitative business 
models are known as predatory publishing, where emerging publications provide a seemingly legitimate website and metrics in order to entice authors to use their services, with the hope of quick financial gain without adequately reviewing the work they publish [4]. In addition to posing a threat to the integrity of legitimate scientific literature [5], predatory publishers have inadvertently created challenges for new, but legitimate journals or publishers with open-access journal offerings, making it more difficult for them to differentiate themselves from the predatory ones [6]. A few days prior to the publication of this protocol, leading authors published a comment in Nature presenting the first consensus definition of "predatory journals and publishers" following a three-round modified Delphi survey involving leading scholars and publishers from ten countries [7]. They defined predatory journals and publishers as "entities that prioritize self-interest at the expense of scholarship and are characterized by false or misleading information, deviation from best editorial and publication practices, a lack of transparency, and/or the use of aggressive and indiscriminate solicitation practices" [7]. This published protocol thus comes at a timely moment; the authors titled their article "Predatory journals: No definition, no defence". The current protocol may provide part of the solution.

High quality research journals typically target a specific niche, clearly define their scope (including the journal's name), and rely on a highly qualified editorial team comprised of researchers who have a track record of publishing peer-reviewed research reflecting the journal's scope [8]. In that context, it is often the reputation of the journal and of its associated editorial team that attract higher quality scientific contributions and lead to higher citations and impact factors. It is therefore very challenging for new journals to emerge, especially those dealing with research topics that are less applicable to broader audiences. Generally, they will target a very local or specialized community of researchers, putting out a call for papers either electronically or by print, and, nowadays, using social media platforms to increase awareness of the new journal and its editorial team. Such methods are simple as they require a limited investment of time, energy, or infrastructure, thus making it easy for predatory publishers to replicate such behaviour.

In mid-2018, the Journal of Natural Health Product Research (JNHPR) was created to serve as the first journal to focus on natural health product (NHP) research regardless of sponsoring institution, scientific discipline, or methodology [9]. The JNHPR has recently become the official journal of the Natural Health Product
Research Society of Canada (NHPRS); a learned society representing academic, government and industry researchers in this field [10]. Our editorial team has chosen to take a different, and to our knowledge, unique approach to better connect with potential contributing researchers. As recognized researchers, our editorial team was drawn to better understand the needs, desires, and capacities of our target audience in a more comprehensive way. A database of international researchers that have published NHP-related scholarly literature has thus been initiated and all the necessary information will be collected from public sources and recorded in an organized manner. The information collected will notably include what subject matter the NHP researchers are investigating, in which institutions they evolve, and in which journals they publish. Rather than using the usual awareness methods mentioned above, the present protocol also outlines the use of a survey administered to the researchers sourced from the database, as a key piece of community and stakeholder engagement. Thus, the purpose of the forthcoming study is to create an NHP researcher database, and survey NHP researchers to gain a better understanding of their attitudes and opinions towards open access publishing. This also provides a process that likeminded publishers can adapt to promote non-predatory, proper, and responsible academic publishing.

\section{Methods}

\section{Natural Health Product Researcher Database}

A web-based search will be carried out and focussed on the countries of Canada, USA, and Australia as well as major countries from the region of West Europe (including the following: Austria, Belgium, Bulgaria, Czech Republic, Denmark, England, Finland, France, Germany, Greece, Italy, Ireland, Norway, Netherlands, Poland, Romania, Spain, Sweden, and Switzerland), with the purpose of creating a database containing the majority of English-speaking/publishing NHP researchers globally. Regions (i.e. state, province or territory) from which each Canadian, American, and Australian researcher is affiliated will also be identified. An alphabetical list will be drawn up for each region that contains major universities and research institutions with the help of resources including Wikipedia Category (https://en.wikipedia.org/wiki/Help:Category). This will maximize the chances that a high percentage of NHP researchers will be found and added to our database in each respective region. Our proposed approach involves identifying the website of each university and research institution in our regional alphabetical list and searching 
for and reading through all faculty and researcher profiles. In the case of a dysfunctional faculty webpage, we will also conduct Google searches using the search terms such as "['University Name' and 'Researcher Name']". Preliminary searches pilot-testing this strategy have identified that faculty and researcher lists on most university/research institution websites are accessible without restrictions. Lists restricted to members of a respective university (i.e. only available to their own students and faculty) will not be included in the study database. In reviewing each faculty member/researcher's web profile, evidence will be sought that they are an actual NHP researcher, by looking for keywords relating to: 1) NHPs as per Health Canada's definition [11] as well as 2) the scope of the JNHPR [12].

For each individual determined to be an NHP researcher by these criteria, the following information will be collected: country/region of affiliation, first and last name, university/institutional affiliation, academic/ professional rank, research interests (keywords), email address, and the URL of the web profile. Information regarding (up to) their five most recently published NHP-related research articles will also be collected and will include: date of publication, title of the article, name of publication (journal), article keywords, article DOI, and article type (i.e. primary research, review article, editorial, etc.). As this information may not always be available on a single webpage, NHP researcher's publications will be identified with the aid of Google Scholar, Google Scholar profiles and PubMed. All NHP researchers sourced and added will be cross-referenced against the database to avoid duplication, as it is anticipated that some researchers may be affiliated with more than one university/institution or even country.

\section{Natural Health Product Researcher Survey}

As the NHP researcher database nears completion, a quantitative observational cross-sectional survey was designed, and will be administered with the aim to understand the knowledge, attitudes and opinions of NHP researchers towards open access publishing, as well as toward the JNHPR. The survey is available as Supplementary File 1. Initial inquiry will include capturing researchers' prior understanding and experience with open access journals. Also, the survey will attempt to identify factors that encourage or hinder their decisions to publish in an open access journal, including hypothesized obstacles, such as article processing fees. The study population will consist of English-speaking/ publishing NHP researchers internationally, and recruitment will use an active, snowball sampling method. All NHP researchers found in our database will be the first to be invited to participate in the survey. However, it is acknowledged that many equally well-respected NHP researchers exist in countries outside of the list we used in creating our NHP database. While this is an acknowledged limitation to our study, it was found that most faculty profiles in these countries were largely not in English. Aside from the fact our team lacks the resources to translate these profiles, some of these researchers may not be fluent in English and could therefore find it more difficult to participate in our survey. To address this limitation, however, survey participants will be encouraged to extend invitations to any other NHP researchers internationally who they think may be interested in participating. It is hoped that this will create a snowball effect where one participant will send an invitation to an NHP researcher in a country not included in our database, and consequently that researcher will send invitations to other national colleagues. Once suggestions of new contacts will wane or additional NHP researchers fail to be found, recruitment will cease and the survey will be considered to be complete. This process should reduce bias in collecting responses. Descriptive statistics will be used to analyze our survey; therefore, no specific power calculation is provided. The margin of error will be calculated and reported based on the sample size and response rate obtained. All responses will be collected using SurveyMonkey. Data will be analyzed using Microsoft Excel and IBM SPSS Statistics (Version 25).

Construction of the NHP researcher database began in early-2018 and the development of our survey began in mid-2019. The survey is anticipated to begin in early 2020 . The link to this published protocol will be included in the survey administered to NHP researchers. Preliminary results will be presented at the NHPRS annual conference. Final results, upon this project's completion, will be published in the JNHPR. The STROBE (Strengthening the Reporting of Observational Studies in Epidemiology) Statement, notably its checklist of items that should be included in reports of cross-sectional studies, will be used to inform the reporting of our survey results [13].

\section{Discussion}

The construction of our NHP researcher database will help identify potential survey participants. This, in turn, will highlight the attitudes and opinions of NHP researchers towards open access publishing and the JNHPR, enabling the journal to better adapt to its potential contributors' needs. It is believed that the publication of this study's protocol may be valuable, not only for the NHP researcher community, but for all researchers and publishers across all fields who have an interest 
in establishing a legitimate peer-reviewed journal in an era of open access and predatory publishing.

Historically, the open access publishing model has increasingly been abused by predatory publishers. The latter are characterized by poor quality or unethical editorial and/or publishing practices as well as operating for the sole purpose of profiting from unsuspecting, unscrupulous or early-career authors $[1,4]$. Though predatory publishers may not consider the impact of their unethical behaviour, the consequences are both frightening and great. Emerging research has confirmed that articles published in predatory journals are cited in the legitimate scientific literature that are indexed in reputable academic databases such as PubMed [14, 15]. Clinicians and policy makers rely on the legitimacy and validity of published scientific research in order to make well-informed decisions that can have significant and life-changing impacts on research, professional practice, policy, and society. The quality of these decisions can therefore be directly influenced by the quality of published research.

Specific to the JNHPR, this survey should provide valuable direction to improve its publication services and better cater to researchers' needs and expectations. This is especially pertinent given the broad scope that the JNHPR seeks to solidly establish. Indeed, research surrounding NHPs continues to grow rapidly and the JNHPR has identified a gap whereby no central publication exists to connect NHP researchers internationally. The results of our survey will shed light on the current profile of NHP researchers worldwide, including their research priorities and relationship with open access publishing. An obvious and acknowledged benefit of the survey will be informing NHP researchers of the JNHPR and of our team's willingness to listen to and engage with its potential contributors. It is also anticipated (and hoped) that survey participants will become interested in serving as future contributors, such as editors, editorial board members, peer-reviewers, and of course, authors. It is acknowledged that the data collected in the planned survey concerns only academic settings and does not capture industry or government research. This limitation will be overcome in future studies through different means, in order to collect data from NHP researchers based in non-academic institutions.

\section{Conclusion}

Open access publishing models are more common now than ever, however, researchers and publishers have a collective responsibility to use this model ethically and be vigilant of how they are abused by predatory journals. In this protocol, a process is provided whereby an international NHP researcher database is constructed and a survey is set up with the aim of understanding the attitudes and opinions of NHP researchers towards open access publishing in general and the JNHPR in particular. Taking this route of paying attention to researchers' concerns, needs, and preferences may provide a means to combat predatory publishing behaviour and this protocol may be found to be useful and/or be adapted by other ethical publishers in the future.

\section{Declarations}

\section{List of Abbreviations}

JNHPR: Journal of Natural Health Product Research;

NHP: natural health product;

NHPRS: Natural Health Product Research Society of Canada;

STROBE: Strengthening the Reporting of Observational Studies in Epidemiology.

\section{Ethics Approval and Consent to Participate}

This study was reviewed by the Hamilton Integrated Research Ethics Board and was deemed to be exempt from their oversight.

\section{Consent for Publication}

All authors consent to this manuscript's publication.

\section{Availability of Data and Materials}

All relevant data are included in this manuscript.

\section{Competing Interests}

The authors declare that they have no competing interests.

\section{Funding}

The establishment of the Journal of Natural Health Product Research was funded in part by a grant from the Federal Government of Canada.

\section{Authors' Contributions}

JYN: made substantial contributions to the design of the protocol, pilot-tested the searches for the NHP researcher database, drafted the manuscript, and gave final approval of the version to be published (D) https://orcid.org/0000-0003-0031-5873 
HQ: pilot-tested the searches for the NHP researcher database, revised the manuscript critically, and gave final approval of the version to be published

FA: made substantial contributions to the design of the protocol, revised the manuscript critically, and gave final approval of the version to be published

KC: made substantial contributions to the design of the protocol, revised the manuscript critically, and gave final approval of the version to be published (D) https://orcid.org/0000-0001-7960-6504

PSH: made substantial contributions to the design of the protocol, revised the manuscript critically, and gave final approval of the version to be published

(D) https://orcid.org/0000-0002-8782-5165

\section{Supplementary Files}

Supplementary File 1: NHP Researcher Survey

\section{References}

[1] Laakso M, Welling P, Bukvova $H$, Nyman L, Björk B, Hedlund $T$. The development of open access journal publishing from 1993 to 2009. PLoS One. 2011 Jun;6(6): e20961. https://doi.org/10.1371/journal.pone.0020961

[2] University of Saskatchewan [Internet]. Predatory Publishers: Home [cited 2019 Nov 21]. Available from: https://libguides.usask.ca/predatorypublishers

[3] Richtig G, Berger M, Lange-Asschenfeldt B, Aberer W, Richtig E. Problems and challenges of predatory journals. Journal of the European Academy of Dermatology and Venereology. 2018 Sep;32(9):1441-9. https://doi. org/10.1111/jdv.15039

[4] PUBLISSO [Internet]. Predatory publishing is the "black sheep" of open access journals [cited 2019 Nov 21]. Available from: https://www.publisso.de/en/advice/ publishing-advice-faqs/predatory-publishing/

[5] Harvey HB, Weinstein DF. Predatory publishing: An emerging threat to the medical literature. Academic Medicine. 2017 Feb;92(2):150-1. https://doi. org/10.1097/ACM.0000000000001521
[6] Memon AR. Revisiting the term predatory open access publishing. Journal of Korean Medical Science. 2019 Mar;34(13). https://doi.org/10.3346/jkms.2019.34.e99

[7] Grudniewicz A, Moher D, Cobey KD, Bryson GL, Cukier S, Allen K. et al. Predatory journals: No definition, no defence. Nature. 2019 Dec;576:210-212. https://doi. org/10.1038/d41586-019-03759-y

[8] BMC [Internet]. What does it take to run your own journal [cited 2019 Nov 21]. Available from: https:// blogs.biomedcentral.com/bmcblog/2014/06/17/ what-does-it-take-to-run-your-own-journal/

[9] Cooley K, Pierre HS, Ng JY. Off with a bang! Introducing the Journal of Natural Health Product Research. Journal of Natural Health Product Research. 2019 Jan; 1(1):1-4. https://doi.org/10.33211/jnhpr.4

[10] Natural Health Product Research Society of Canada (NHPRS) [Internet]. Natural Health Product Research Society of Canada [cited 2019 Nov 21]. Available from https://www.nhprs.ca/

[11] Government of Canada [Internet]. Natural health products [cited 2019 Nov 21]. Available from: https://www. canada.ca/en/health-canada/services/drugs-healthproducts/natural-non-prescription.html

[12] Journal of Natural Health Product Research (JNHPR) [Internet]. Aims and Scope [cited 2019 Nov 21]. Available from: https://jnhpresearch.com/index.php/ jnhpr/about/\#_Toc487899578

[13] Von Elm E, Altman DG, Egger M, Pocock SJ, Gøtzsche PC, Vandenbroucke JP. The Strengthening the Reporting of Observational Studies in Epidemiology (STROBE) statement: guidelines for reporting observational studies. Annals of Internal Medicine. 2007 Oct;147(8):573-7. https://doi. org/10.7326/0003-4819-147-8-200710160-00010

[14] Severin A, Low N. Readers beware! Predatory journals are infiltrating citation databases. International Journal of Public Health. 2019;64:1123-1124. https://doi.org/ 10.1007/s00038-019-01284-3

[15] Ross-White A, Godfrey CM, Sears KA, Wilson R. Predatory publications in evidence syntheses. Journal of the Medical Library Association: JMLA. 2019 Jan;107(1):57. https://doi.org/10.5195\%2Fjmla.2019.491 


\section{Article Information}

Managing Editor: Pierre Haddad, Kieran Cooley

This study was reviewed by the Hamilton Integrated Research Ethics Board and was deemed to be exempt from their oversight.

Article Dates: Published Dec 2019

\section{Citation}

Please cite this article as follows:

Ng JY, Quach H, Ashbury FD, Cooley K, Haddad PS. Favouring responsible publishing: A protocol for creating a database of researchers and surveying their knowledge, attitudes and opinions towards open access publishing and a new field-specific journal. Journal of Natural Health Product Research. 201912 20: 1(2).

https://jnhpresearch.com/index.php/jnhpr/article/view/9

DOI Link: https://doi.org/10.33211/jnhpr.9

\section{Copyright}

(c) Jeremy Y Ng, Halton Quach, Fredrick D. Ashbury, Kieran Cooley, Pierre S Haddad. (2019). Published first in the Journal of Natural Health Product Research. This is an open access article distributed under the terms of the Creative Commons Attribution License (https://creativecommons.org/licenses/by/4.0/), which permits unrestricted use, distribution, and reproduction in any medium, provided the original work, first published in the Journal of Natural Health Product Research, an NHP Publications journal, is properly cited. The complete bibliographic information, a link to the original publication on https://www.jnhpresearch.com, as well as this copyright and license information must be included.

Journal of Natural Health Product Research
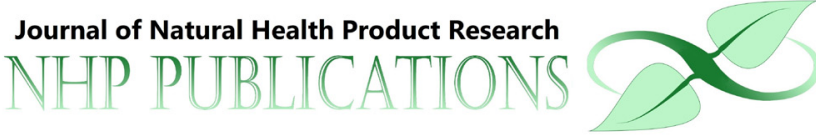

Canadà

Does the safety, efficacy, and quality of natural health products matter to YOU? Submit your research article to the Journal of Natural Health Product Research!

Pre-submission inquiries? Send us an email at editorial.office@jnhpresearch.com Facebook, Twitter and LinkedIn: @NHPPublications 